Universidad Católica del Norte

Antofagasta - Chile

\title{
Laplacian integral graphs with a given degree sequence constraint
}

\author{
Anderson Fernandes Novanta \\ CEFET-RJ, Brasil \\ Carla Silva Oliveira \\ ENCE/IBGE, Brasil \\ and \\ Leonardo de Lima \\ UFPR, Brasil \\ Received : February 2021. Accepted : May 2021
}

\begin{abstract}
Let $G$ be a graph on $n$ vertices. The Laplacian matrix of $G$, denoted by $L(G)$, is defined as $L(G)=D(G)-A(G)$, where $A(G)$ is the adjacency matrix of $G$ and $D(G)$ is the diagonal matrix of the vertex degrees of $G$. A graph $G$ is said to be $L$-integral if all eigenvalues of the matrix $L(G)$ are integers. In this paper, we characterize all $L$ integral non-bipartite graphs among all connected graphs with at most two vertices of degree larger than or equal to three.
\end{abstract}

Keywords: Laplacian matrix; Eigenvalues; L-integral graphs.

Mathematics Subject Classifications: $A M S$ 15A15, $15 F 10$.

*Partially supported by grants 314298/2018-5 and 304548/2020-0, CNPq, Brazil. 


\section{Introduction}

Let $G=(V, E)$ be an undirected graph, without loops or multiple edges. Let $d(G)=\left(d_{1}(G), d_{2}(G), \ldots, d_{n}(G)\right)$ be the sequence degree of $G$, such that $\Delta(G)=d_{1}(G) \geq d_{2}(G) \geq \cdots \geq \delta(G)=d_{n}(G)$. The vertex connectivity of $G, k(G)$, is the minimum number of vertices that need to be removed such that the graph $G$ gets disconnected and $P_{n}$ is a path with $n$ vertices. We define $\mathcal{G}_{1}$ as the family of connected graphs with sequence degree in a way that $d_{1} \geq 3$ and $1 \leq d_{i} \leq 2, i=2, \ldots, n$. Also, we define $\mathcal{G}_{2}$ as the family of connected graphs with sequence degree such that $d_{1} \geq d_{2} \geq 3$ and $1 \leq d_{i} \leq 2, i=3, \ldots, n$. The sum graph, denoted by $G=G_{1}+G_{2}$, is the graph $G$ such that $V=V_{1} \times V_{2}$ and each pair of vertices $\left(u_{1}, u_{2}\right)$ and $\left(v_{1}, v_{2}\right)$ are adjacent in $G$ if and only if $u_{1}=v_{1}$ and $\left(u_{2}, v_{2}\right) \in E_{2}$ or $u_{2}=v_{2}$ and $\left(u_{1}, v_{1}\right) \in E_{1}$. The Firefly graph, denoted by $F_{r, s, t}$, is the graph with $2 r+s+2 t+1$ vertices that contains $r$ triangles, $s$ pendant edges and $t$ pendant paths of length 2 sharing a common vertex. We write $A(G)$ for the $(0,1)$-adjacency matrix of a graph and $D(G)$ for the diagonal matrix of the vertex degrees of $G$. Also, we write $L(G)=D(G)-A(G)$ and $Q(G)=D(G)+A(G)$ for the Laplacian matrix and signless Laplacian matrix of $G$. A graph $G$ is $L$-integral (resp. $Q$-integral) if all of its $L$-eigenvalues (resp. $Q$-eigenvalues) are integers. The spectrum of the Laplacian matrix of $G$ is denoted by $\operatorname{Spec}_{L}(G)=$ $\left\{\mu_{1}(G)^{\left[n_{1}\right]}, \mu_{2}(G)^{\left[n_{2}\right]}, \ldots, \mu_{s}(G)^{\left[n_{s}\right]}\right\}$, where $\mu_{i}(G)$ is the $i$-th largest Laplacian eigenvalue and $n_{i}$ is its algebraic multiplicity. The algebraic connectivity of $G$ is denoted by $a(G)=\mu_{n-1}(G)$.

In 1994, Grone and Merris [3] initiated the study of the $L$-integral graph and Merris in [11] presented an explicit construction of all maximal graphs which are $L$-integral. After this, some infinite families of $L$-integral graphs were characterized in the literature as it can be seen in $[8,9,10,11,12]$. In particular, Kirkland in [9] determined all Laplacian integral graphs such that $\Delta(G)=3$. Motivated by that, we study all Laplacian integral graphs with at most two vertices of degree greater than or equal to 3 . It is worth mentioning that Novanta et al. in [13] found all bipartite $Q$-integral graphs in $\mathcal{G}_{1}$ and $\mathcal{G}_{2}$. Since for bipartite graphs the $L$ - and $Q$-eigenvalues coincide all $L$-integral bipartite graphs within those families are determined. In this paper, we determine all non bipartite $L$-integral graphs in the families $\mathcal{G}_{1}$ and $\mathcal{G}_{2}$. Thus, we state our main result:

Theorem 1. Let $G$ be a graph on $n \geq 9$ vertices with at most two vertices of degree greater than two. Then $G$ is L-integral if and only if $G$ is one of 
the following: $K_{1, n-1}, K_{2}+K_{1, n-3}, K_{2, n-2}, F_{r, s, 0}$, where $s \geq 1$ and $r \geq 1$, $K_{1} \vee\left(r K_{1} \cup s K_{2} \cup K_{1, t}\right)$, where $t \geq 2$ and $r+s \geq 2$ or $K_{2} \vee(n-2) K_{1}$.

The remaining content of the paper is organized as follows. In Section 2 , we will give some important results that will be needed in the sequel. In Section 3 , we present all non bipartite $L$-integral graphs in the family $\mathcal{G}_{1}$. In Section 4, we present all non bipartite $L$-integral graphs in the family $\mathcal{G}_{2}$. In this paper, we use the same demonstration techniques presented in [13].

\section{Preliminaries}

In this section, we present some results that will be useful to prove the main results of the paper.

Lemma 1. Let $G$ be a connected graph. Then $a(G)>0$.

Lemma 2. Let $G$ be a non-complete graph. Then $a(G) \leq k(G) \leq \delta(G)$.

Theorem 3. Let $G$ be a non-complete and connected graph on $n$ vertices. Then $k(G)=a(G)$ if only if $G$ can be written as $G=G_{a} \vee G_{b}$, where $G_{a}$ is a disconnected graph on $(n-k(G))$ vertices and $G_{b}$ is a graph on $k(G)$ vertices with $a\left(G_{b}\right) \geq 2 k(G)-n$.

Lemma 4. Let $A$ be a block diagonal matrix whith diagonal blokcs $A_{i i}$, for $1 \leq i \leq k$ are blocks of $A$. Then, $\operatorname{det} A=\prod_{i=1}^{k} \operatorname{det} A_{i i}$.

Defintion 5. Given a graph $G$, and a matrix $M=\left[m_{i j}\right]$ associated with $G$, a partition $\pi$ of $V(G), V(G)=V_{1} \cup \cdots \cup V_{k}$ is equitable with respect to $G$ and $M$, if for all $i, j \in\{1,2, \cdots, k\}$

$$
\sum_{t \in V_{j}} m_{s t}=d_{i j}
$$

is a constant $d_{i j}$ for any $s \in V_{i}$.

The matrix $M_{\pi}=\left[d_{i j}\right]$ of order $k$ is called the divisor matrix of $M$ associated with the partition $\pi$.

Theorem 6. Any eigenvalue of $M_{\pi}$ is also an eigenvalue of $M$. 
Lemma 7. Let $G$ be a graph on $n$ vertices and $f$ an edge of $G$. If $H \cong G \backslash f$ then

$$
\mu_{1}(G) \geq \mu_{1}(H) \geq \mu_{2}(G) \geq \mu_{2}(H) \geq \ldots \geq \mu_{n}(G) \geq \mu_{n}(H) .
$$

If $H$ is a subgraph of $G$ obtained by removing $r$ edges, then for each $i=$ $1, \ldots, n-r$.

$$
\mu_{i}(G) \geq \mu_{i}(H) \geq \mu_{i+r}(G) .
$$

Let $A$ be a matrix of order $n$ and $1 \leq r \leq n$. The matrix $A_{r}$ of order $r$ is a principal submatrix of $A$ obtained by deleting $n-r$ rows and the corresponding columns from $A$.

Proposition 8. Let $A$ be a Hermitian matrix of order $n$, let $r$ be an integer with $1 \leq r<n$, and let $A_{r}$ be a principal submatrix of $A$ of order $r$ with eigenvalues $\lambda_{1} \geq \cdots \geq \lambda_{n}$ and $\theta_{1} \geq \cdots \geq \theta_{r}$ respectively. Then, for each $i=1, \ldots, r$

$$
\lambda_{i} \geq \theta_{i} \geq \lambda_{i+n-r}
$$

Remark 9. Let $B_{n-2}$ be a principal submatrix of $L(G)$ of order $n-2$. From Proposition 8 , we have that $\theta_{n-3}\left(B_{n-2}\right) \geq \mu_{n-1}(G)$. If $G$ is connected and $B_{n-2}$ has at least two eigenvalues in the interval $(0,1)$, we conclude that $0<\mu_{n-1}(G)<1$.

Remark 10. For $n \geq 7$, there are at least 2 eigenvalues of $L\left(P_{n}\right)$ in the interval $(0,1)$.

Consider a family of $p$ graphs, $\mathcal{F}=\left\{G_{1}, \cdots, G_{p}\right\}$, where each graph $G_{j}$ has order $n_{j}$, for $j=1, \cdots, p$, and a graph $H$ such that $V(H)=\{1, \cdots, p\}$. Each vertex $j \in V(H)$ is assigned to the graph $G_{j} \in \mathcal{F}$. The $H-j$ oin (generalized composition) of $G_{1}, \cdots, G_{p}$ is the graph $G=H\left[G_{1}, \cdots, G_{p}\right]$ such that $V(G)=\bigcup_{j=1}^{p} V\left(G_{j}\right)$ and $E(G)=\left(\bigcup_{j=1}^{p} V\left(E_{j}\right)\right) \cup\left(\bigcup_{r s \in E(H)}\{u v\right.$ : $\left.\left.u \in V\left(G_{r}\right), v \in V\left(G_{s}\right)\right\}\right)$.

Theorem 11. Let $G$ be a graph on $n$ vertices with at most two vertices of degree greater than or equal to 3. Then $G$ is $Q$-integral if and only if $G$ is one of the following: $K_{1, n-1}, K_{2}+K_{1, n-3}, K_{2, n-2}$ or $P_{4}\left[K_{2}, K_{1}, K_{1}, K_{2}\right]$.

The following results characterize cographs from forbidden $P_{4}$ and show that all cographs are $L$-integral.

Theorem 12. A graph is cograph if and only if it does not have an induced subgraph isomorphic to $P_{4}$.

Theorem 13. If $G$ is cograph then $G$ is a $L$-integral. 


\section{L-integral graphs in $\mathcal{G}_{1}$}

In [13], Novanta et al. characterized all $L$-integral bipartite graphs belonging to $\mathcal{G}_{1}$. In this section, we characterize all $L$-integral non-bipartite graphs in $\mathcal{G}_{1}$. The graphs that belong to family $\mathcal{G}_{1}$ are graphs that contain cycles, paths and pending vertices with one vertex, say $u$, in common such that $d(u) \geq 3$. Notice that the Firefly graphs, $F_{r, s, t}$, belong to the family $\mathcal{G}_{1}$. In particular, $F_{0, n-1,0} \cong K_{1, n-1}, F_{0,0,1} \cong K_{1,2}$ are $L$-integral graphs. Below, we present the main result of this section.

Theorem 1. Let $G \in \mathcal{G}_{1}$ be a graph on $n$ vertices. Then, $G$ is L-integral if only if either $G \cong K_{1, n-1}$ or $G \cong F_{r, s, 0}$, with $s \geq 1$ and $r \geq 1$.

Proof. Let $G \in \mathcal{G}_{1}$. If $G$ is bipartite, from Theorem 11, $G$ is $L$-integral if and only if $G \cong K_{1, n-1}$. Now, suppose that $G$ is non-bipartite and $L$ integral. From Lemmas 1 and $2,0<a(G) \leq k(G)=1$, and consequently $a(G)=1$. From Theorem $3, G \cong G_{a} \vee G_{b}$ where $V\left(G_{b}\right)=\{u\}$. As $G \in \mathcal{G}_{1}$ and for any $x \in V(G), d(x) \leq 2$, we have that $G_{a} \cong r \cdot K_{1} \cup s \cdot K_{2}$ where $r \geq 1$ and $s \geq 1$. So, $G \cong F_{r, s, 0}$ which is a cograph. From Theorem 13, $G$ is $L$ - integral and the result follows.

\section{L-integral graphs in $\mathcal{G}_{2}$}

In [13], Novanta et al. characterized all $L$-integral bipartite graphs belonging to $\mathcal{G}_{2}$. Let $\mathcal{G}_{2}^{\prime}$ be the subfamily of non-bipartite graphs belonging to $\mathcal{G}_{2}$. From Lemmas 1 and 2, we have that $0<a(G) \leq k(G) \leq 2$. So, in order to characterize all $L$-integral graphs in $\mathcal{G}_{2}^{\prime}$ we need to consider the cases: $a(G)=k(G)$ and $a(G)<k(G)$.

Case 1: $G \in \mathcal{G}_{2}^{\prime}$ and $a(G)=k(G)$.

Theorem 1. Let $G \in \mathcal{G}_{2}^{\prime}$ with $n \geq 7$ vertices. Then $G$ is $L$-integral if and only if $G \cong K_{1} \vee\left(r \cdot K_{1} \cup s \cdot K_{2} \cup K_{1, t}\right)$, where $t \geq 2$ and $r+s \geq 2$ or $G \cong K_{2} \vee(n-2) \cdot K_{1}$. 
Proof. Let $G \in \mathcal{G}_{2}^{\prime}$ with $n \geq 7$ vertices. Suppose that $G$ is $L$-integral. So, $a(G)=k(G)=1$ or $a(G)=k(G)=2$. Firstly, suppose that $a(G)=$ $k(G)=1$. From Theorem $3, G \cong G_{a} \vee G_{b}$ where $V\left(G_{b}\right)=\{u\}$ and, consequently, $v \in V\left(G_{a}\right)$. Let $x \in V\left(G_{a}\right)$ such that $x \neq v$. As $G \in \mathcal{G}_{2}$, $d(x) \leq 2$, we conclude that $G_{a} \cong r \cdot K_{1} \cup s \cdot K_{2} \cup K_{1, t}$, where $t \geq 2$ and $r+s \geq 2$. Now, suppose that $a(G)=k(G)=2$. From Theorem 3, $G \cong G_{a} \vee G_{b}$ such that $G_{b}$ is a graph on two vertices. So, $u, v \in V\left(G_{b}\right)$ and $G_{b} \cong K_{2}$. Let $x \in V\left(G_{a}\right)$. As $G \in \mathcal{G}_{2}^{\prime}$ and $d(x) \leq 2$, we conclude that $G_{a} \cong(n-2) \cdot K_{1}$. Then, $G \cong K_{2} \vee(n-2) \cdot K_{1}$ and the result follows.

Case 2: $G \in \mathcal{G}_{2}^{\prime}$ and $a(G)<k(G)$.

In this case, we characterize all $L$-integral graphs in $\mathcal{G}_{2}^{\prime}$ such that $a(G)<k(G) \leq 2$. If $k(G)=1, G$ is not $L$-integral. So, we only need to consider that $k(G)=2$. Then, $G$ has only cycles that contain two vertices $u$ and $v$ of degree larger than or equal to 3 . Consequently, we need to analyze the length of paths with end vertices $u$ and $v$.

Proposition 2. Let $G \in \mathcal{G}_{2}^{\prime}$ with $n \geq 11$ vertices. If $G$ has a subgraph $P_{k}$, for $k \geq 9$, with end vertices $u$ and $v$, then $G$ is not $L$-integral.

Proof. Let $G \in \mathcal{G}_{2}^{\prime}$ with $n \geq 11$ vertices. For $k \geq 9$, suppose that $G$ contains a path $P_{k}$ with sequence of vertices $u x_{1} \cdots x_{k-2} v$. Let $H$ be the subgraph of $G$ obtained by removing the edges $u x_{1}$ and $x_{k-2} v$. So, $H \cong$ $H_{1} \cup P_{k-2}$, where $H_{1}$ is a non bipartite graph and $\mu_{n}(H)=\mu_{n-1}(H)=0$. From Remark 10, $P_{k-2}$ has at least 2 eigenvalues in the interval $(0,1)$. Then, we assume that $0<\mu_{n-2}(H) \leq \mu_{n-3}(H)<1$. From Lemma 7 , we conclude that $0<\mu_{n-1}(G) \leq \mu_{n-3}(H)<1$. Therefore, $G$ is not $L$-integral.

From Proposition 2 (Section 4), now we need to consider the remaining cases when $G$ has a subgraph $P_{k}$ for $3 \leq k \leq 8$. First, we consider that $G \in \mathcal{G}_{2}^{\prime}$ is a graph that contains $r$ paths $P_{n_{p}}^{p}$, for $1 \leq p \leq \Delta(G)=\Delta$ with the sequences of vertices $u x_{1}^{p} \cdots x_{n_{p}-2}^{p} v$ such that $n_{p} \in\{3,5,7\}$. As $G$ is non bipartite graph, the vertices $u$ and $v$ should be adjacent. By a convenient labeling for the vertices, $L(G)$ can be seen written in the following way:

$L(G)=\left[\begin{array}{ccccc}\mathcal{D}_{2 \times 2} & \mathcal{T}_{2 \times\left(n_{1}-2\right)} & \mathcal{T}_{2 \times\left(n_{2}-2\right)} & \cdots & \mathcal{T}_{2 \times\left(n_{\Delta}-2\right)} \\ \mathcal{T}_{\left(n_{1}-2\right) \times 2} & \mathcal{A}_{n_{1}-2} & 0_{\left(n_{1}-2\right) \times\left(n_{2}-2\right)} & \cdots & 0_{\left(n_{1}-2\right) \times\left(n_{\Delta}-2\right)} \\ \mathcal{T}_{\left(n_{2}-2\right) \times 2} & 0_{\left(n_{2}-2\right) \times\left(n_{2}-2\right)} & \mathcal{A}_{\left(n_{2}-2\right)} & \cdots & 0_{\left(n_{2}-2\right) \times\left(n_{\Delta}-2\right)} \\ \vdots & \vdots & \vdots & \ddots & \vdots \\ \mathcal{T}_{\left(n_{\Delta}-2\right) \times 2} & 0_{\left(n_{\Delta}-2\right) \times\left(n_{1}-2\right)} & 0_{\left(n_{\Delta}-2\right) \times\left(n_{2}-2\right)} & \cdots & \mathcal{A}_{n_{\Delta}-2}\end{array}\right](I)$, 
where $\mathcal{D}=\left[d_{i j}\right]_{2 \times 2}$ such that $d_{i j}=\left\{\begin{array}{cc}\Delta, & \text { if } i=j \\ -1, & \text { if } i \neq j\end{array} \quad\right.$ and $\mathcal{T}=$ $\left[t_{i j}\right]_{2 \times n_{p-2}}$ such that $t_{i j}=\left\{\begin{array}{c}-1, i=j=1 \\ -1, i=2 \text { and } j=n_{p}-2 \\ 0, \text { otherwise. }\end{array}\right.$

Observe that $A_{n_{p}-2} \in\left\{A_{1}, A_{3}, A_{5}\right\}$, where

$$
A_{1}=[2], A_{3}=\left[\begin{array}{ccc}
2 & -1 & 0 \\
-1 & 2 & -1 \\
0 & -1 & 2
\end{array}\right] \text { and } A_{5}=\left[\begin{array}{ccccc}
2 & -1 & 0 & 0 & 0 \\
-1 & 2 & -1 & 0 & 0 \\
0 & -1 & 2 & -1 & 0 \\
0 & 0 & -1 & 2 & -1 \\
0 & 0 & 0 & -1 & 2
\end{array}\right] \text {. }
$$

Notice that since $G$ is non-bipartite, the vertices $u$ and $v$ should be adjacent and we will use this fact to prove Proposition 3,4 and 5 .

Proposition 3. Let $G \in \mathcal{G}_{2}^{\prime}$ with $n \geq 9$ vertices. If $G$ has at least two subgraphs $P_{5}$, or at least two subgraphs $P_{7}$ or one subgraph $P_{5}$ together with a subgraph $P_{7}$ with end vertices $u$ and $v$, then $G$ is not $L$-integral.

Proof. Let $G \in \mathcal{G}_{2}^{\prime}$ with $n \geq 9$ vertices. Suppose that $G$ contains at least two paths $P_{5}$, or at least two paths $P_{7}$ or one path $P_{5}$ together with a path $P_{7}$ with the sequence of vertices $u x_{1}^{i} \cdots x_{n_{p}-2}^{i} v$ such that $n_{p} \in\{5,7\}$ and $i \geq 2$. In all cases, let $B_{n-2}$ be the principal submatrix of $L(G)$ obtained by removing both rows and columns that correspond to the vertices $u$ and $v$. It is easy to see that $B_{n-2}$ is a block diagonal matrix and its blocks are the matrices $A_{3}$ and/or $A_{5}$ which have eigenvalues in the interval $(0,1)$. From Proposition 8 , we have $0<\mu_{n-1}(G) \leq \theta_{n-3}\left(B_{n-2}\right)<1$. Then, $G$ is not $L$-integral.

As $G$ is a non-bipartite graph, the following remaining cases are described as: (i) $G$ has at least one subgraph $P_{3}$ and one subgraph $P_{5}$, and (ii) $G$ has at least one subgraph $P_{3}$ and one subgraph $P_{7}$. Next, Proposition 4 proves case (i), and Proposition 5 proves case (ii).

Proposition 4. Let $G \in \mathcal{G}_{2}^{\prime}$ with $n \geq 6$ vertices. If $G$ has $s \geq 1$ subgraphs $P_{3}$ and one subgraph $P_{5}$ with end vertices $u$ and $v$, then $G$ is not $L$-integral.

Proof. Let $G \in \mathcal{G}_{2}^{\prime}$ with $n \geq 6$ vertices. Recall that $u v \in G$. It is easy to see that $G$ is not $L$-integral graph for $s=1$. Suppose that $s \geq 2$. From matrix $(I), L(G)$ can be seen written in the following way: 


$$
L(G)=\left[\begin{array}{cccccc}
\mathcal{D}_{2 \times 2} & \mathcal{T}_{2 \times 1} & \mathcal{T}_{2 \times 1} & \cdots & \mathcal{T}_{2 \times 1} & \mathcal{T}_{2 \times 3} \\
\mathcal{T}_{1 \times 2} & \mathcal{A}_{1} & 0_{1 \times 1} & \cdots & 0_{1 \times 1} & 0_{1 \times 3} \\
\mathcal{T}_{1 \times 2} & 0_{1 \times 1} & \mathcal{A}_{1} & \cdots & 0_{1 \times 1} & 0_{1 \times 3} \\
\vdots & \vdots & \vdots & \ddots & \vdots & \vdots \\
\mathcal{T}_{1 \times 2} & 0_{1 \times 1} & 0_{1 \times 1} & \cdots & \mathcal{A}_{1} & 0_{1 \times 3} \\
\mathcal{T}_{3 \times 2} & 0_{3 \times 1} & 0_{3 \times 1} & \cdots & 0_{3 \times 1} & \mathcal{A}_{3}
\end{array}\right]
$$

where $\Delta=s+2$. According to Theorem 6 , the eigenvalues of the matrix

$$
R_{L(G)}=\left[\begin{array}{cccccc}
s+2 & -1 & -s & -1 & 0 & 0 \\
-1 & s+2 & -s & 0 & 0 & -1 \\
-1 & -1 & 2 & 0 & 0 & 0 \\
-1 & 0 & 0 & 2 & -1 & 0 \\
0 & 0 & 0 & -1 & 2 & -1 \\
0 & -1 & 0 & 0 & -1 & 2
\end{array}\right]
$$

are eigenvalues of $L(G)$, whose characteristic polynomial is $p(\lambda)=\lambda^{6}+$ $(-2 s-12) \lambda^{5}+\left(s^{2}+18 s+55\right) \lambda^{4}+\left(-6 s^{2}-56 s-120\right) \lambda^{3}+\left(10 s^{2}+70 s+\right.$ $125) \lambda^{2}+\left(-4 s^{2}-30 s-50\right) \lambda$. As $p(1)=s^{2}-1>0$, for $s \geq 2$, and $p(2)=-4 s<0$, we conclude that there is a root in the interval $(1,2)$, and consequently $G$ is not $L$-integral.

Proposition 5. Let $G \in \mathcal{G}_{2}^{\prime}$ with $n \geq 8$ vertices. If $G$ has at least $s \geq 1$ subgraphs $P_{3}$ and one subgraph $P_{7}$ with end vertices $u$ and $v$, then $G$ is not $L$-integral.

Proof. Let $G \in \mathcal{G}_{2}^{\prime}$ with $n \geq 8$ vertices. It is easy to see that $G$ is not $L$-integral for $s=1$. Suppose that $s \geq 2$. From matrix $(I), L(G)$ has the following form:

$$
L(G)=\left[\begin{array}{cccccc}
\mathcal{D}_{2 \times 2} & \mathcal{T}_{2 \times 1} & \mathcal{T}_{2 \times 1} & \cdots & \mathcal{T}_{2 \times 1} & \mathcal{T}_{2 \times 5} \\
\mathcal{T}_{1 \times 2} & \mathcal{A}_{1} & 0_{1 \times 1} & \cdots & 0_{1 \times 1} & 0_{1 \times 5} \\
\mathcal{T}_{1 \times 2} & 0_{1 \times 1} & \mathcal{A}_{1} & \cdots & 0_{1 \times 1} & 0_{1 \times 5} \\
\vdots & \vdots & \vdots & \ddots & \vdots & \vdots \\
\mathcal{T}_{1 \times 2} & 0_{1 \times 1} & 0_{1 \times 1} & \cdots & \mathcal{A}_{1} & 0_{1 \times 5} \\
\mathcal{T}_{5 \times 2} & 0_{5 \times 1} & 0_{5 \times 1} & \cdots & 0_{5 \times 1} & \mathcal{A}_{5}
\end{array}\right],
$$

where $\Delta=s+2$. Applying the Intermediate Value Theorem to the characteristic polynomial associated to matrix obtained by Theorem 6 for the 
matrix $L(G)$, we conclude that there is a root in the interval $(3,4)$, and consequently $G$ is not $L$-integral.

Remark 6. If $G \in \mathcal{G}_{2}^{\prime}$ and $G$ has only subgraph $P_{3}$ with end vertices $u$ and $v, a(G)=k(G)$ which was analyzed in Case 4.1.

Now let us analyze the cases in which $G \in \mathcal{G}_{2}^{\prime}$ is a graph that contains $r$ paths $P_{n_{p}}^{p}$ with the sequence of vertices $u x_{1}^{p} \cdots x_{n_{p}-2}^{p} v$, for $1 \leq p \leq r$, and $n_{p} \in\{4,6,8\}$. By a convenient labeling to the vertices of $G$ we obtain

$L(G)=\left[\begin{array}{ccccc}\mathcal{D}_{2 \times 2} & \mathcal{T}_{2 \times\left(n_{1}-2\right)} & \mathcal{T}_{2 \times\left(n_{2}-2\right)} & \cdots & \mathcal{T}_{2 \times\left(n_{r}-2\right)} \\ \mathcal{T}_{\left(n_{1}-2\right) \times 2} & \mathcal{A}_{n_{1}-2} & 0_{\left(n_{1}-2\right) \times\left(n_{2}-2\right)} & \cdots & 0_{\left(n_{1}-2\right) \times\left(n_{r}-2\right)} \\ \mathcal{T}_{\left(n_{2}-2\right) \times 2} & 0_{\left(n_{2}-2\right) \times\left(n_{2}-2\right)} & \mathcal{A}_{\left(n_{2}-2\right)} & \cdots & 0_{\left(n_{2}-2\right) \times\left(n_{r}-2\right)} \\ \vdots & \vdots & \vdots & \ddots & \vdots \\ \mathcal{T}_{\left(n_{r}-2\right) \times 2} & 0_{\left(n_{r}-2\right) \times\left(n_{1}-2\right)} & 0_{\left(n_{r}-2\right) \times\left(n_{2}-2\right)} & \cdots & \mathcal{A}_{n_{r}-2}\end{array}\right](I I)$,

where

$\mathcal{D}=\left[d_{i j}\right]_{2 \times 2}$ such that $d_{i j}=\left\{\begin{array}{cl}\Delta, & \text { if } i=j \\ -1, & \text { if } i \neq j \\ 0, & \text { if } i \neq j \text { and } u \sim v \text { and } u \nsim v\end{array}\right.$ and $\mathcal{T}=$ $\left[t_{i j}\right]_{2 \times n_{p}-2}$ such that $t_{i j}=\left\{\begin{array}{cc}1, & \text { if } i=j=1, \\ -1, & \text { if } i=2 \text { and } j=n_{p}-2, \\ 0, & \text { otherwise. }\end{array}\right.$

Observe that $A_{n_{p}-2} \in\left\{A_{2}, A_{4}, A_{6}\right\}$, where

$A_{2}=\left[\begin{array}{cc}2 & -1 \\ -1 & 2\end{array}\right]$,

$A_{4}=\left[\begin{array}{cccc}2 & -1 & 0 & 0 \\ -1 & 2 & -1 & 0 \\ 0 & -1 & 2 & -1 \\ 0 & 0 & -1 & 2\end{array}\right]$ and $A_{6}=\left[\begin{array}{cccccc}2 & -1 & 0 & 0 & 0 & 0 \\ -1 & 2 & -1 & 0 & 0 & 0 \\ 0 & 1 & 2 & 1 & 0 & 0 \\ 0 & 0 & -1 & 2 & -1 & 0 \\ 0 & 0 & 0 & -1 & 2 & -1 \\ 0 & 0 & 0 & 0 & -1 & 2\end{array}\right]$.

By using the matrix $L(G)$ presented above, we obtain the following propositions.

Proposition 7. Let $G \in \mathcal{G}_{2}^{\prime}$ with $n \geq 9$ vertices. If $G$ has a subgraph $P_{8}$ with end vertices $u$ and $v$, then $G$ is not $L$-integral. 
Proof. Let $G \in \mathcal{G}_{2}^{\prime}$ with $n \geq 9$ vertices. Suppose that $G$ contains a path $P_{8}$ with the sequence of vertices $u x_{1} \cdots x_{6} v$. Let $B_{n-2}$ be the principal submatrix of $L(G)$ obtained by removing both rows and columns that correspond to vertices $u$ and $v$. Note that $B_{n-2}$ is a block diagonal matrix and one of its blocks is the matrix $A_{6}$, which has two eigenvalues in the interval $(0,1)$. From Lemma 4 , we have $0<\theta_{n-2}\left(B_{n-2}\right)<\theta_{n-3}\left(B_{n-2}\right)<1$ and consequently from Proposition 8 , we conclude that $0<\mu_{n-1}(G)=$ $a(G) \leq \theta_{n-3}\left(B_{n-2}\right)<1$. Therefore, $G$ is not $L$-integral.

Proposition 8. Let $G \in \mathcal{G}_{2}^{\prime}$ with $n \geq 7$ vertices. If $G$ has a subgraph $P_{6}$ with end vertices $u$ and $v$, then $G$ is not L-integral.

Proof. Let $G \in \mathcal{G}_{2}^{\prime}$ with $n \geq 7$ vertices. Suppose that $G$ contains $a \geq 2$ paths $P_{6}$ with the sequence of vertices $u x_{1}{ }^{i} \cdots x_{4}{ }^{i} v$ for $2 \leq i \leq a$. Let $B_{n-2}$ be the principal submatrix of $L(G)$ obtained by removing both rows and columns that correspond to vertices $u$ and $v$. Note that $B_{n-2}$ is a block diagonal matrix and $a \geq 2$ of its blocks is the matrix $A_{4}$. It is easy to see that $A_{4}$ has one eigenvalue in the interval $(0,1)$. Then, $B_{n-2}$ contains $a \geq 2$ eigenvalues in the interval $(0,1)$. From Lemma 4 , we have $0<\theta_{n-2}\left(B_{n-2}\right)<\theta_{n-3}\left(B_{n-2}\right)<1$, and consequently, from Proposition 8 , we conclude that $0<\mu_{n-1}(G) \leq \theta_{n-3}\left(B_{n-2}\right)<1$. Therefore, $G$ is not $L$-integral. Now, suppose that $G$ contains one path $P_{6}$ with the sequence of vertices $u x_{1} \cdots x_{4} v$ along with one path $P_{5}$ or one path $P_{7}$. Therefore the principal submatrix of $L(G)$ obtained by removing both rows and columns that correspond to vertices $u$ and $v, B_{n-2}$, is a block diagonal matrix wich has at least two blocks $A_{6}$ and $A_{5}$ or $A_{6}$ and $A_{7}$. In both cases, $B_{n-2}$ contains $a \geq 2$ eigenvalues in the interval $(0,1)$ and, consequently, from Proposition 8, we conclude that $0<\mu_{n-1}(G) \leq \theta_{n-3}\left(B_{n-2}\right)<1$. Then, $G$ is not $L$-integral. Finally, suppose that $G$ contains one path $P_{6}$, with the sequence of vertices $u x_{1} \cdots x_{4} v$, with $s \geq 1$ paths $P_{3}$ and $t \geq 0$ path $P_{4}$. So, we need to consider the following cases:

Case 1: $G$ contains $t \geq 1$ paths $P_{4}, s \geq 1$ paths $P_{3}$ and one path $P_{6}$.

Case 1.1: $u$ and $v$ are adjacent.

By a convenient labeling for the vertices, $L(G)$ can be seen represented in the following way: 


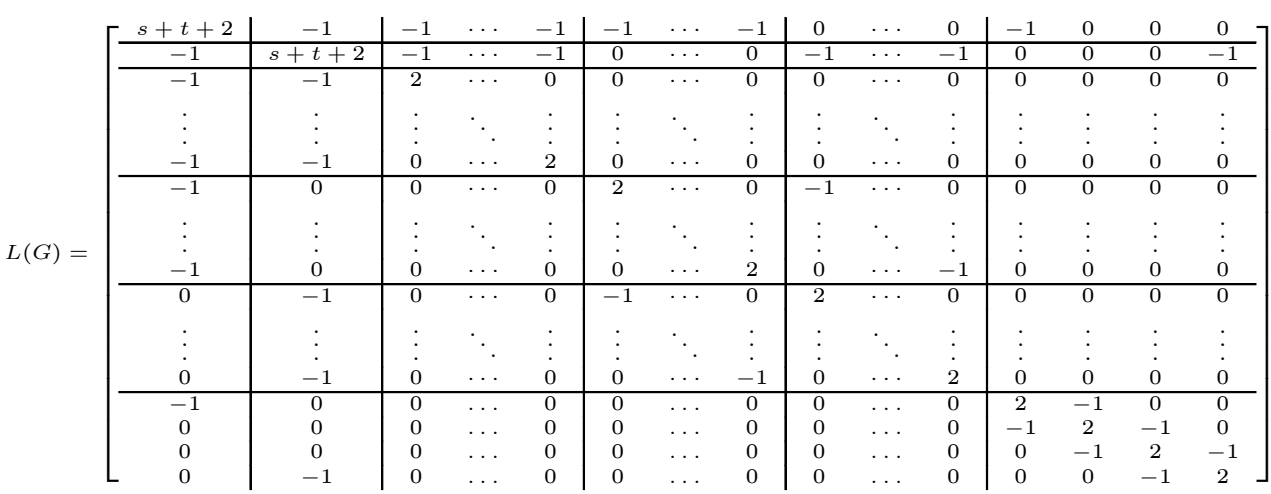

Applying the Intermediate Value Theorem to the characteristic polynomial associated to matrix obtained by Theorem 6 for the matrix $L(G)$, we conclude that there is a root in the interval $(3,4)$, and consequently $G$ is not $L$-integral.

Case 1.2: $u$ and $v$ are non-adjacent.

By a convenient labeling for the vertices, $L(G)$ can be seen written in the following way:

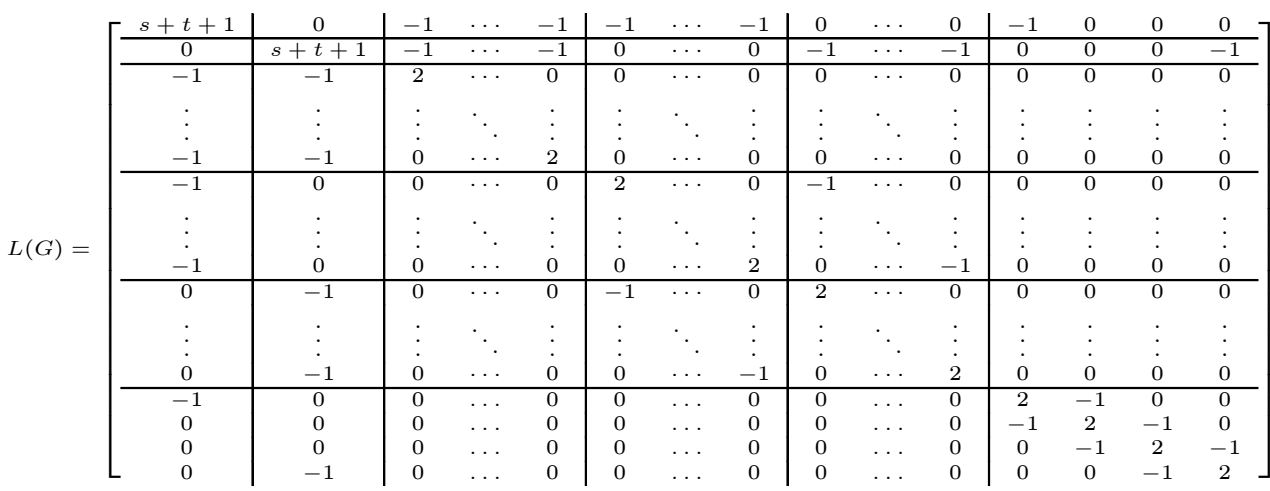

Applying the same technique as in Case 1.1, we conclude that there is a root in the interval $(3,4)$, and consequently $G$ is not $L$-integral.

Case 2: $G$ contains $s \geq 1$ paths $P_{3}$ and one path $P_{6}$.

Case 2.1: $u$ and $v$ are adjacent.

From the matrix $(I I), L(G)$ can be seen written in the following way: 


$$
L(G)=\left[\begin{array}{cccccc}
\mathcal{D}_{2 \times 2} & \mathcal{T}_{2 \times 1} & \mathcal{T}_{2 \times 1} & \cdots & \mathcal{T}_{2 \times 1} & \mathcal{T}_{2 \times 4} \\
\mathcal{T}_{1 \times 2} & \mathcal{A}_{1} & 0_{1 \times 1} & \cdots & 0_{1 \times 1} & 0_{1 \times 4} \\
\mathcal{T}_{1 \times 2} & 0_{1 \times 1} & \mathcal{A}_{1} & \cdots & 0_{1 \times 1} & 0_{1 \times 4} \\
\vdots & \vdots & \vdots & \ddots & \vdots & \vdots \\
\mathcal{T}_{1 \times 2} & 0_{1 \times 1} & 0_{1 \times 1} & \cdots & \mathcal{A}_{1} & 0_{1 \times 4} \\
\mathcal{T}_{4 \times 2} & 0_{4 \times 1} & 0_{4 \times 1} & \cdots & 0_{4 \times 1} & \mathcal{A}_{4}
\end{array}\right]
$$

where $\Delta=s+2$. Applying the same technique as in Case 1.1, we conclude that there is a root in the interval $(1,2)$, and consequently $G$ is not $L$ integral.

Case 2.2: $u$ and $v$ are non-adjacent.

It is easy to see that for $s=1$ or $s=2, G$ is not $L$-integral. Suppose that $s \geq 3$. From the matrix $(I I), L(G)$ can be seen written in the following way:

$$
L(G)=\left[\begin{array}{cccccc}
\mathcal{D}_{2 \times 2} & \mathcal{T}_{2 \times 1} & \mathcal{T}_{2 \times 1} & \cdots & \mathcal{T}_{2 \times 1} & \mathcal{T}_{2 \times 4} \\
\mathcal{T}_{1 \times 2} & \mathcal{A}_{1} & 0_{1 \times 1} & \cdots & 0_{1 \times 1} & 0_{1 \times 4} \\
\mathcal{T}_{1 \times 2} & 0_{1 \times 1} & \mathcal{A}_{1} & \cdots & 0_{1 \times 1} & 0_{1 \times 4} \\
\vdots & \vdots & \vdots & \ddots & \vdots & \vdots \\
\mathcal{T}_{1 \times 2} & 0_{1 \times 1} & 0_{1 \times 1} & \cdots & \mathcal{A}_{1} & 0_{1 \times 4} \\
\mathcal{T}_{4 \times 2} & 0_{4 \times 1} & 0_{4 \times 1} & \cdots & 0_{4 \times 1} & \mathcal{A}_{4}
\end{array}\right]
$$

where $\Delta=s+1$. Applying the same technique as in Case 1.1, we conclude that there is a root in the interval $(2,3)$, and consequently $G$ is not $L$ integral.

Proposition 9. Let $G \in \mathcal{G}_{2}^{\prime}$ with $n \geq 6$ vertices. If $G$ has a subgraph $P_{4}$ with end vertices $u$ and $v$, then $G$ is not $L$-integral.

Proof. Let $G \in \mathcal{G}_{2}^{\prime}$ with $n \geq 6$ vertices. Suppose that $G$ contains one path $P_{4}$ with the sequence of vertices $u x_{1} x_{2} v$ and with at least two paths in the set $\left\{P_{5}, P_{7}\right\}$. Let $B_{n-2}$ be the submatrix principal of $L(G)$ obtained by removing boths rows and columns corresponding to vertices $u$ and $v$. As $B_{n-2}$ is a block diagonal matrix and its blocks belong to the set $\left\{A_{3}\right.$, $\left.A_{5}\right\}$ which have one eigenvalue in the interval $(0,1)$, from Proposition 8 (Section 2), we conclude that $0<\mu_{n-1}(G) \leq \theta_{n-3}\left(B_{n-2}\right)<1$. Then, $G$ is not $L$-integral. Now, suppose that $G$ contains $t \geq 1$ paths $P_{4}$, with the sequence of vertices $u x_{1}^{q} x_{2}^{q} v$, such that $1 \leq q \leq t, s \geq 1$ paths $P_{3}$ or/and one path of the set $\left\{P_{5}, P_{7}\right\}$. So we need to analyse the following cases: 
Case 1: $G$ contains $t \geq 1$ paths $P_{4}$ and $s \geq 1$ paths $P_{3}$.

Case 1.1: $u$ and $v$ are adjacent.

By a convenient labeling for the vertices, $L(G)$ can be seen written in the following way:

$\left[\begin{array}{c|c|ccc|ccc|ccc}s+t+1 & -1 & -1 & \cdots & -1 & -1 & \cdots & -1 & 0 & \cdots & 0 \\ \hline-1 & s+t+1 & -1 & \cdots & -1 & 0 & \cdots & 0 & -1 & \cdots & -1 \\ \hline-1 & -1 & 2 & \cdots & 0 & 0 & \cdots & 0 & 0 & \cdots & 0 \\ \vdots & \vdots & \vdots & \ddots & \vdots & \vdots & \ddots & \vdots & \vdots & \ddots & \vdots \\ -1 & -1 & 0 & \cdots & 2 & 0 & \cdots & 0 & 0 & \cdots & 0 \\ \hline-1 & 0 & 0 & \cdots & 0 & 2 & \cdots & 0 & -1 & \cdots & 0 \\ \vdots & \vdots & \vdots & \ddots & \vdots & \vdots & \ddots & \vdots & \vdots & \ddots & \vdots \\ -1 & 0 & 0 & \cdots & 0 & 0 & \cdots & 2 & 0 & \cdots & -1 \\ \hline 0 & -1 & 0 & \cdots & 0 & -1 & \cdots & 0 & 2 & \cdots & 0 \\ \vdots & \vdots & \vdots & \ddots & \vdots & \vdots & \ddots & \vdots & \vdots & \ddots & \vdots \\ 0 & -1 & 0 & \cdots & 0 & 0 & \cdots & -1 & 0 & \cdots & 2\end{array}\right]$.

Applying the Intermediate Value Theorem to the characteristic polynomial associated to matrix obtained by Theorem 6 for the matrix $L(G)$, we conclude that there is a root in the interval $(1,2)$, and, consequently, $G$ is not $L$-integral.

Case 1.2: $u$ and $v$ are non-adjacent.

By a convenient labeling for the vertices, $L(G)$ can be seen written in the following way:

$$
L(G)=\left[\begin{array}{c|c|ccc|ccc|ccc}
s+t & 0 & -1 & \cdots & -1 & -1 & \cdots & -1 & 0 & \cdots & 0 \\
\hline 0 & s+t & -1 & \cdots & -1 & 0 & \cdots & 0 & -1 & \cdots & -1 \\
\hline-1 & -1 & 2 & \cdots & 0 & 0 & \cdots & 0 & 0 & \cdots & 0 \\
\vdots & \vdots & \vdots & \ddots & \vdots & \vdots & \ddots & \vdots & \vdots & \ddots & \vdots \\
-1 & -1 & 0 & \cdots & 2 & 0 & \cdots & 0 & 0 & \cdots & 0 \\
\hline-1 & 0 & 0 & \cdots & 0 & 2 & \cdots & 0 & -1 & \cdots & 0 \\
\vdots & \vdots & \vdots & \ddots & \vdots & \vdots & \ddots & \vdots & \vdots & \ddots & \vdots \\
-1 & 0 & 0 & \cdots & 0 & 0 & \cdots & 2 & 0 & \cdots & -1 \\
\hline 0 & -1 & 0 & \cdots & 0 & -1 & \cdots & 0 & 2 & \cdots & 0 \\
\vdots & \vdots & \vdots & \ddots & \vdots & \vdots & \ddots & \vdots & \vdots & \ddots & \vdots \\
0 & -1 & 0 & \cdots & 0 & 0 & \cdots & -1 & 0 & \cdots & 2
\end{array}\right] .
$$


According to Theorem 6, the eigenvalues of the matrix

$$
R_{L(G)}=\left[\begin{array}{ccccc}
s+t & 0 & -s & -t & 0 \\
0 & s+t & -s & 0 & -t \\
-1 & -1 & 2 & 0 & 0 \\
-1 & 0 & 0 & 2 & -1 \\
0 & -1 & 0 & -1 & 2
\end{array}\right]
$$

are eigenvalues of $L(G)$, whose characteristic polynomial is $p(\lambda)=\lambda^{5}+$ $(-2 s-2 t-6) \lambda^{4}+\left(s^{2}+2 s t+t^{2}+10 s+10 t+11\right) \lambda^{3}+\left(-4 s^{2}-8 s t-4 t^{2}-\right.$ $14 s-14 t-6) \lambda^{2}+\left(3 s^{2}+8 s t+4 t^{2}+6 s+4 t\right) \lambda$. Then, we have:

(i) for $s=1$ and $t \geq 2, p(\lambda)=\lambda\left(\lambda^{2}-\lambda(4+t)+3+2 t\right)^{2}$, whose roots are $0, \frac{-\sqrt{t^{2}+4}+t+4}{2}$ with multiplicity 2 , and $\frac{\sqrt{t^{2}+4}+t+4}{2}$ with multiplicity 2 as well. As $t<\sqrt{t^{2}+4}<t+1, p(\lambda)$ has non-integer roots ;

(ii) for $s=2$ and $t=1, \operatorname{Spec}_{L}(G)=\left\{4.73^{[1]}, 4^{[1]}, 2^{[2]}, 1.27^{[1]}, 0^{[1]}\right\}$;

(iii) for $s=2$ and $t=2, \operatorname{Spec}_{L}(G)=\left\{5.56^{[1]}, 5^{[1]}, 3^{[1]}, 2^{[2]}, 1.44^{[1]}, 1^{[1]}, 0^{[1]}\right\}$;

(iv) for $s=2$ and $t \geq 3, \quad p(\lambda)=(-2 t-10) \lambda^{4}+\lambda^{5}+\left(t^{2}+14 t+\right.$ $35) \lambda^{3}+\left(-4 t^{2}-30 t-50\right) \lambda^{2}+\left(4 t^{2}+20 t+24\right) \lambda$, whose roots are $0,2, t+3, \frac{-\sqrt{t^{2}+2 t+9}+t+5}{2}, \frac{\sqrt{t^{2}+2 t+9}+t+5}{2}$. As $t+1<\sqrt{t^{2}+2 t+9}<$ $t+2, p(\lambda)$ has non-integer roots ;

(v) for $s \geq 3$ and $t \geq 1, p(2)=-2 s^{2}+4 s<0$ and $p(3)=6 s t+3 t^{2}-6 t>0$. So, we conclude that there is a root in the interval $(2,3)$.

Therefore, in all previous cases we obtain that $G$ is not $L$-integral.

Case 2: $G$ contains $t \geq 1$ paths $P_{4}, s \geq 0$ paths $P_{3}$, and one path $P_{5}$.

Case 2.1: $u$ and $v$ are adjacent. By a convenient labeling for the vertices, $L(G)$ can be seen written in the following way:

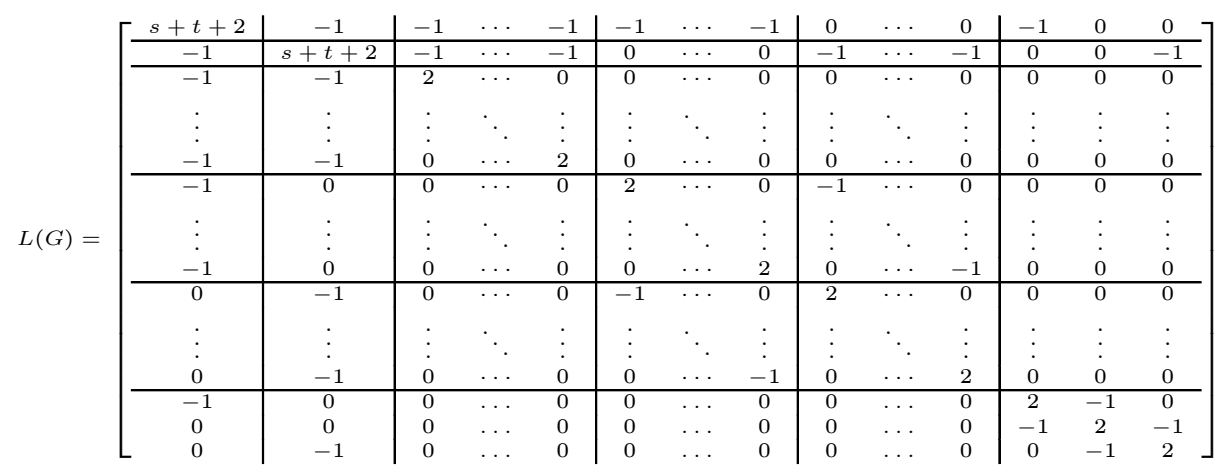


Applying the Intermediate Value Theorem to the characteristic polynomial associated to matrix obtained by Theorem 6 for the matrix $L(G)$, we conclude that there is a root in the interval $(0.5,1)$, and consequently $G$ is not $L$-integral.

Case 2.2: $u$ and $v$ are non-adjacent.

For $s=0$ and $t=2$, it is easy to see that $G$ is not $L$-integral. By a convenient labeling for the vertices, $L(G)$ can be seen written in the following way:

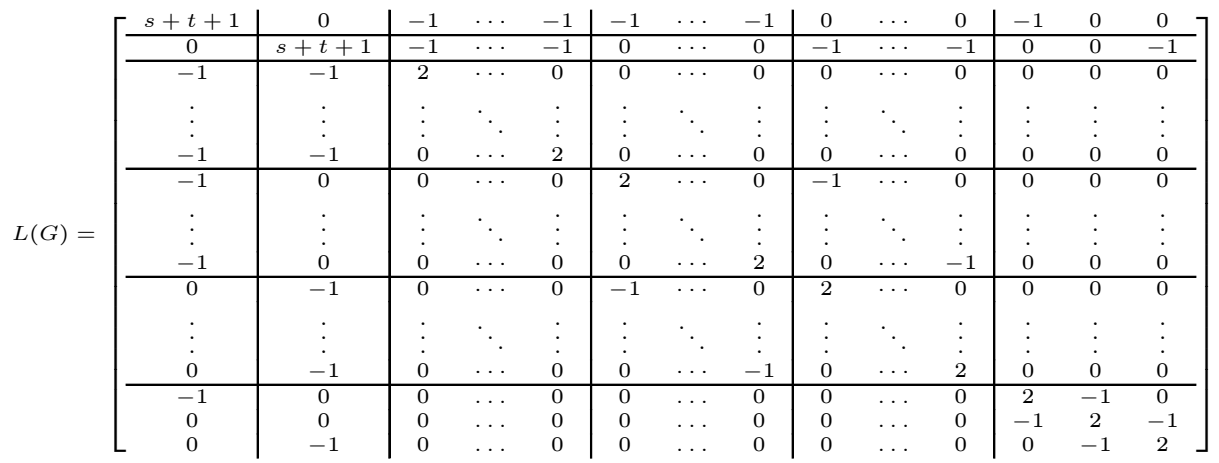

Applying the same technique as in Case 2.1, we conclude that there is a root in the interval $(0.5,1)$, and consequently $G$ is not $L$-integral.

Case 3: $G$ contains $t \geq 1$ paths $P_{4}, s \geq 1$ paths $P_{3}$ and one path $P_{7}$.

Case 3.1: $u$ and $v$ are adjacent.

By a convenient labeling for the vertices, $L(G)$ can be seen written in the following way:

$\left[\begin{array}{c|c|ccc|ccc|ccc|ccccc}s+t+2 & -1 & -1 & \cdots & -1 & -1 & \cdots & -1 & 0 & \cdots & 0 & -1 & 0 & 0 & 0 & 0 \\ \hline-1 & s+t+2 & -1 & \cdots & -1 & 0 & \cdots & 0 & -1 & \cdots & -1 & 0 & 0 & 0 & 0 & -1 \\ \hline-1 & -1 & 2 & \cdots & 0 & 0 & \cdots & 0 & 0 & \cdots & 0 & 0 & 0 & 0 & 0 & 0 \\ \vdots & \vdots & \vdots & \ddots & \vdots & \vdots & \ddots & \vdots & \vdots & \ddots & \vdots & \vdots & \vdots & \vdots & \vdots & \vdots \\ -1 & -1 & 0 & \cdots & 2 & 0 & \cdots & 0 & 0 & \cdots & 0 & 0 & 0 & 0 & 0 & 0 \\ \hline-1 & 0 & 0 & \cdots & 0 & 2 & \cdots & 0 & -1 & \cdots & 0 & 0 & 0 & 0 & 0 & 0 \\ \vdots & \vdots & \vdots & \ddots & \vdots & \vdots & \ddots & \vdots & \vdots & \ddots & \vdots & \vdots & \vdots & \vdots & \vdots & \vdots \\ -1 & 0 & 0 & \cdots & 0 & 0 & \cdots & 2 & 0 & \cdots & -1 & 0 & 0 & 0 & 0 & 0 \\ \hline 0 & -1 & 0 & \cdots & 0 & -1 & \cdots & 0 & 2 & \cdots & 0 & 0 & 0 & 0 & 0 & 0 \\ \vdots & \vdots & \vdots & \ddots & \vdots & \vdots & \ddots & \vdots & \vdots & \ddots & \vdots & \vdots & \vdots & \vdots & \vdots & \vdots \\ 0 & -1 & 0 & \cdots & 0 & 0 & \cdots & -1 & 0 & \cdots & 2 & 0 & 0 & 0 & 0 & 0 \\ \hline-1 & 0 & 0 & \cdots & 0 & 0 & \cdots & 0 & 0 & \cdots & 0 & 2 & -1 & 0 & 0 & 0 \\ 0 & 0 & 0 & \cdots & 0 & 0 & \cdots & 0 & 0 & \cdots & 0 & -1 & 2 & -1 & 0 & 0 \\ 0 & 0 & 0 & \cdots & 0 & 0 & \cdots & 0 & 0 & \cdots & 0 & 0 & -1 & 2 & -1 & 0 \\ 0 & 0 & 0 & \cdots & 0 & 0 & \cdots & 0 & 0 & \cdots & 0 & 0 & 0 & -1 & 2 & -1 \\ 0 & -1 & 0 & \cdots & 0 & 0 & \cdots & 0 & 0 & \cdots & 0 & 0 & 0 & 0 & -1 & 2\end{array}\right]$


Applying the same technique as in Case 2.1, we conclude that there is a root in the interval $(0.5,1)$, and consequently $G$ is not $L$-integral.

Case 3.2: $u$ and $v$ are not adjacent.

By a convenient labeling for the vertices, $L(G)$ can be seen written in the following way:

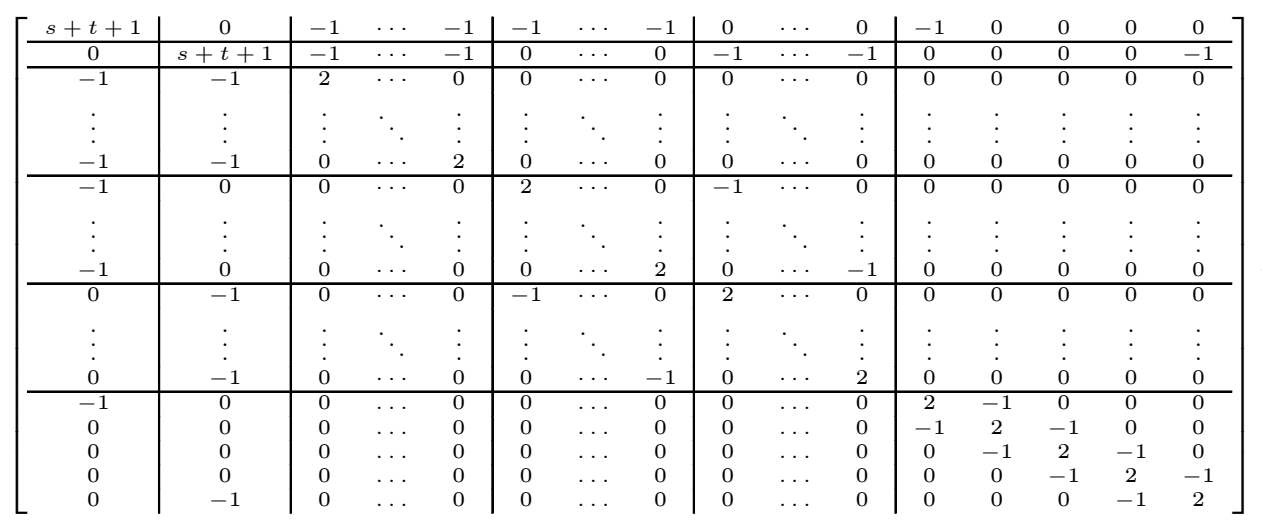

Applying the same technique as in Case 2.1, we conclude that there is a root in the interval $(0.5,1)$, and consequently $G$ is not $L$-integral.

Theorem 10. Let $G \in \mathcal{G}_{2}^{\prime}$ with $n \geq 9$ vertices. Then $G$ is $L$-integral if and only if $G \cong K_{1} \vee\left(r \cdot K_{1} \cup s \cdot K_{2} \cup K_{1, t}\right)$, where $t \geq 2$ and $r+s \geq 2$ or $G \cong K_{2} \vee(n-2) \cdot K_{1}$.

Proof. Let $G \in \mathcal{G}_{2}^{\prime}$ with $n \geq 9$ vertices. Suppose that $G$ is $L$-integral. From Theorem 1 (Section 4) and Propositions 2, 3, 4, 5, 7, 8 and 9 (Section 4) we conclude that $G \cong K_{1} \vee\left(r \cdot K_{1} \cup s \cdot K_{2} \cup K_{1, t}\right)$, where $t \geq 2$ and $r+s \geq 2$ or $G \cong K_{2} \vee(n-2) \cdot K_{1}$ and the result follows.

By Theorems 11 (Section 2), 1 (Section 3) and 10 (Section 4), the proof of Theorem 1 (Section 1) is complete. 


\section{References}

[1] A. E. Brouwer and W. H. Haemers, Spectra of graphs. New York,NY: Springer, 2012.

[2] M. Fiedler. "Algebraic connectivity of graphs", Czechoslovak mathematical journal, vol. 23, pp. 298-305, 1973.

[3] R. Grone and R. Merris, "The laplacian spectrum of a graph", SIAM Journal discrete mathematics, vol. 7, pp. 221-229, 1994.

[4] J. van den Heuvel, "Hamilton cycles and eigenvalues of graphs", Linear algebra and its applications, vol. 226-228, pp. 723-730, 1995.

[5] R. A. Horn and C. R. Johnson, Matrix analysis, 2nd ed. Cambridge: Cambridge University Press, 2013.

[6] P. Hof and D. Paulusma, "A new characterization of P6-free graphs", Discrete applied mathematics, vol. 158, pp. 731-740, 2010.

[7] S. J. Kirkland, J. J. Molitierno, M. Neumann, and B. L. Shader, "On graphs with equal algebraic and vertex connectivity", Linear algebra and its applications, vol. 341, pp. 45-56, 2002.

[8] S. J. Kirkland, "Constructably laplacian integral graphs", Linear algebra and its applications, vol. 423, pp. 3-21, 2007.

[9] S. J. Kirkland, "Laplacian integral graphs with maximum degree 3", The electronic journal of combinatorics, vol. 15, Art. ID. 120, 2008.

[10] L. S. de Lima, N. M. de Abreu, and C. S. Oliveira, "Laplacian integral graphs in S(a,b)", Linear algebra and its applications, vol. 423, pp. 136-145, 2007.

[11] R. Merris, "Degree maximal graphs are laplacian integral", Linear algebra and its applications, vol. 199, pp. 381-389, 1994.

[12] R. Merris, "Laplacian graph eigenvectores", Linear algebra and its applications, vol. 278, pp. 221-236, 1998.

[13] A. F. Novanta, L. S. de Lima, and C. S. Oliveira, "Q-integral graphs with at most two vertices of degree greater than or equal to three", Linear algebra and its applications, vol. 614, pp. 144-163, 2021. 


\section{Anderson Fernandes Novanta}

Programa de Pós Graduação em Engenharia de Produção e Sistemas, Centro Federal de Educação Tecnológica Celso Suckow da Fonseca (CEFET-RJ),

Rio de Janeiro,

Brasil

e-mail: anderson novanta@yahoo.com.br

\section{Carla Silva Oliveira}

Escola Nacional de Ciências Estatísticas (ENCE/IBGE),

Rio de Janeiro,

Brasil

e-mail: carla.oliveira@ibge.gov.br

Corresponding author

and

\section{Leonardo de Lima}

Universidade Federal do Parana (UFPR),

Curitiba, Parana,

Brasil

e-mail: leonardo.delima@ufpr.br 